\title{
High-efficiency and low-loss gallium nitride dielectric metasurfaces for nanophotonics at visible wavelengths
}

Naresh Kumar Emani, Egor Khaidarov, Ramón Paniagua-Domínguez, Yuan Hsing Fu, Vytautas Valuckas, Shunpeng Lu, Xueliang Zhang, Swee Tiam Tan, Hilmi Volkan Demir, and Arseniy I. Kuznetsov

Citation: Appl. Phys. Lett. 111, 221101 (2017); doi: 10.1063/1.5007007

View online: https://doi.org/10.1063/1.5007007

View Table of Contents: http://aip.scitation.org/toc/apl/111/22

Published by the American Institute of Physics

\section{Articles you may be interested in}

Dense arrays of site-controlled quantum dots with tailored emission wavelength: Growth mechanisms and optical properties

Applied Physics Letters 111, 221102 (2017); 10.1063/1.5004407

Dual-surface flexible $\mathrm{THz}$ Fano metasensor

Applied Physics Letters 111, 201101 (2017); 10.1063/1.5000428

Characterization of an active metasurface using terahertz ellipsometry

Applied Physics Letters 111, 191101 (2017); 10.1063/1.5004194

Spontaneous emission enhancement of colloidal perovskite nanocrystals by a photonic crystal cavity

Applied Physics Letters 111, 221104 (2017); 10.1063/1.5000248

Control of light polarization by voltage in excitonic metasurface devices

Applied Physics Letters 111, 241101 (2017); 10.1063/1.5005827

$234 \mathrm{~nm}$ and $246 \mathrm{~nm}$ AIN-Delta-GaN quantum well deep ultraviolet light-emitting diodes

Applied Physics Letters 112, 011101 (2018); 10.1063/1.5007835

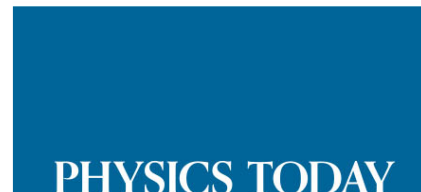

WHITEPAPERS
MANAGER'S GUIDE

Accelerate R\&D with

Multiphysics Simulation
READ NOW

PRESENTED BY

И๐ $\subset$ MSOL 


\title{
High-efficiency and low-loss gallium nitride dielectric metasurfaces for nanophotonics at visible wavelengths
}

\author{
Naresh Kumar Emani, ${ }^{1, a), b)}$ Egor Khaidarov, ${ }^{1,2, a)}$ Ramón Paniagua-Domínguez, ${ }^{1}$ \\ Yuan Hsing Fu, ${ }^{1}$ Vytautas Valuckas, ${ }^{1}$ Shunpeng Lu, ${ }^{2}$ Xueliang Zhang, ${ }^{2}$ Swee Tiam Tan, ${ }^{2}$ \\ Hilmi Volkan Demir, ${ }^{2,3, \mathrm{c})}$ and Arseniy I. Kuznetsov ${ }^{1, \mathrm{c})}$ \\ ${ }^{1}$ Data Storage Institute, A*STAR (Agency for Science, Technology and Research), 2 Fusionopolis Way, \\ \#08-01 Innovis, Singapore 138634 \\ ${ }^{2}$ LUMINOUS! Center of Excellence for Semiconductor Lighting and Displays, The Photonics Institute, \\ School of Electrical and Electronic Engineering, Nanyang Technological University, 50 Nanyang Avenue, \\ Singapore 639798 \\ ${ }^{3}$ Department of Electrical and Electronic Engineering, Department of Physics, UNAM - The National \\ Nanotechnology Research Center and Institute of Materials Science and Nanotechnology, Bilkent University, \\ Bilkent, Ankara 06800, Turkey
}

(Received 29 September 2017; accepted 4 November 2017; published online 27 November 2017)

\begin{abstract}
The dielectric nanophotonics research community is currently exploring transparent material platforms (e.g., $\mathrm{TiO}_{2}, \mathrm{Si}_{3} \mathrm{~N}_{4}$, and $\mathrm{GaP}$ ) to realize compact high efficiency optical devices at visible wavelengths. Efficient visible-light operation is key to integrating atomic quantum systems for future quantum computing. Gallium nitride ( $\mathrm{GaN})$, a III-V semiconductor which is highly transparent at visible wavelengths, is a promising material choice for active, nonlinear, and quantum nanophotonic applications. Here, we present the design and experimental realization of high efficiency beam deflecting and polarization beam splitting metasurfaces consisting of GaN nanostructures etched on the $\mathrm{GaN}$ epitaxial substrate itself. We demonstrate a polarization insensitive beam deflecting metasurface with $64 \%$ and $90 \%$ absolute and relative efficiencies. Further, a polarization beam splitter with an extinction ratio of 8.6/1 (6.2/1) and a transmission of $73 \%$ (67\%) for p-polarization (s-polarization) is implemented to demonstrate the broad functionality that can be realized on this platform. The metasurfaces in our work exhibit a broadband response in the blue wavelength range of $430-470 \mathrm{~nm}$. This nanophotonic platform of GaN shows the way to off- and on-chip nonlinear and quantum photonic devices working efficiently at blue emission wavelengths common to many atomic quantum emitters such as $\mathrm{Ca}^{+}$and $\mathrm{Sr}^{+}$ions. Published by AIP Publishing. https://doi.org/10.1063/1.5007007
\end{abstract}

Metasurfaces have emerged as a highly promising approach to realize compact nanophotonic devices including phase masks, ${ }^{1}$ waveplates, ${ }^{2}$ focusing lenses, ${ }^{3}$ focal plane arrays, ${ }^{4}$ flat mirrors, ${ }^{5}$ and holograms. ${ }^{6}$ Most of the early studies on metasurfaces were based on thin plasmonic nanoantenna arrays arranged in various permutations and combinations. ${ }^{7,8}$ Plasmonic metasurfaces enable light manipulation with ultrathin devices, but they suffer significant ohmic losses which degrade the performance of all plasmonic devices. This is fundamentally due to the fact that the electromagnetic energy is stored as kinetic energy of electrons for one-half of the optical cycle. ${ }^{9}$ On the other hand, in the past couple of years, dielectric metasurfaces have gained increasing prominence essentially because of the small optical loss in dielectrics at frequencies below their bandgaps as well as the capability of high index dielectric materials to support both electric and magnetic resonances in nanostructures, which offers a richer design space. ${ }^{10}$ To date, the dielectric metasurface research community has predominantly focused on developing the design concepts based on resonant antennas, ${ }^{10,11}$ Pancharatnam-Berry phase, ${ }^{12-15}$ and waveguide

\footnotetext{
${ }^{a)}$ N. K. Emani and E. Khaidarov contributed equally to this work.

${ }^{b}$ Currently at Indian Institute of Technology, Hyderabad, India.

${ }^{c)}$ Authors to whom correspondence should be addressed: volkan@stanfordalumni.org and arseniy_k@dsi.a-star.edu.sg
}

approaches ${ }^{16-19}$ to improve the efficiency of nanophotonic devices. Interestingly, the use of high index dielectrics to design subwavelength gratings has been investigated almost two decades earlier. We refer to an excellent recent review by Lalanne and Chavel for a comprehensive historical background on the dielectric approach to metalenses. ${ }^{20}$

A survey of the dielectric metasurface literature also reveals that silicon, more specifically amorphous $\mathrm{Si}$, has been extensively used primarily because of its wellestablished nanofabrication processes. However, $\mathrm{Si}$ is not a good material choice at visible wavelengths because of its strong intrinsic absorption. Wide bandgap dielectrics such as $\mathrm{TiO}_{2}{ }^{15,17,21}$ and $\mathrm{Si}_{3} \mathrm{~N}_{4},{ }^{22}$ which are transparent at visible wavelengths, are currently being investigated as potential low-loss alternatives. The materials discussed thus far are all passive and hence are not suitable for active applications where optical gain is necessary. Direct bandgap III-V materials are very promising for such active applications because of their strong dipole transition strength and smaller free carrier lifetimes compared to indirect bandgap materials. Typically, the crystal structure of III-V materials does not possess centrosymmetry, and hence, they exhibit large second order susceptibility $\left(\chi^{2}\right)$, which can be exploited to realize optically switchable nonlinear devices. Indeed, recently, GaAs-based high aspect ratio nanostructures have been used 
to demonstrate optical switching of metasurfaces. ${ }^{23-25}$ Even though GaAs is a good material choice for both nonlinear response and emission at near-IR wavelengths, it cannot be applied at visible wavelengths due to its high optical losses. Another potential alternative is $\mathrm{GaP}$, which was shown to be an effectively loss-less platform for dielectric metasurfaces above $560 \mathrm{~nm} \cdot{ }^{26}$ Efficient blue wavelength operation is of critical importance for on-chip quantum and nonlinear optics with color-centers and atomic transitions. ${ }^{27}$ In this paper, we experimentally demonstrate epitaxial GaN on sapphire, which is a material of immense technological interest for solid-state lighting technologies, as a viable platform for metasurfaces at visible wavelengths. Thanks to its high transparency through the whole visible spectrum, relatively high refractive index ( $>2.4$ in the visible), and well-developed industrial use as an active material for blue-, cyan-, and green-emitting LEDs and lasers for general lighting, backlighting, and other applications, this platform may pave the way for applications of dielectric metasurfaces to nonlinear and quantum optics. Indeed, III-Nitride materials have already been used to demonstrate electrically driven, ${ }^{28}$ room-temperature ${ }^{29}$ single photon emission. Here, we experimentally show a high-efficiency beam deflecting metasurface and a polarization-splitting metasurface as examples of the viability of $\mathrm{GaN}$ as a platform for nanophotonics. The metasurfaces were realized on top of epitaxial GaN on sapphire wafer by etching the nanostructures directly into the epitaxial GaN layer. Very recently, first demonstrations of GaN based focusing lenses with a transmissivity of $\sim 86 \%$ for blue wavelength operation have been published. $^{30,31}$ In these examples, GaN nanostructures were fabricated directly on top of a sapphire substrate. In our work, GaN nanoantennas are located on the GaN epitaxy with the same refractive index, which paves the way for a wider range of applications but provides additional design constraints.

The primary building block of our metasurface, optimized for operation at a wavelength of $460 \mathrm{~nm}$, which is a typical emission peak for digital lighting and backlighting, ${ }^{32}$ is a nanopillar of height $460 \mathrm{~nm}$, as schematically illustrated in
Fig. 1(a). Each pillar can be considered as a waveguide which allows certain modes to propagate with an effective mode index defined by the pillar diameter. The phase shift and transmission through the unit cell, which are dependent on the diameter and the height of the nanopillar, were calculated by numerical modeling using the finite difference time domain (FDTD) technique in commercially available Lumerical $^{\mathrm{TM}}$ software. The relative phase accumulated along the nanopillar, with the size of unit cell fixed at $330 \mathrm{~nm}$ in both lateral dimensions and $460 \mathrm{~nm}$ in height, can be seen in Fig. 1(b). The period of the repeating nanopillars was chosen such that the resulting nanopillar array is sub-diffractive (in air) and small enough to achieve sufficient phase sampling while being also large enough to neglect interactions between nanopillars. To verify the hypothesis of non-interacting nanopillars, we calculated the phase delay introduced by $460 \mathrm{~nm}$ length of an isolated long cylinder given by $460 \mathrm{~nm} \times \beta_{H E 11}$, where $\beta_{H E 11}$ is the propagation constant of the fundamental mode in a long cylinder. ${ }^{33}$ This is shown as a dashed line in Fig. 1(b), which closely follows the phase delay estimated by the FDTD simulations, indicating that the phase shift introduced by the nanopillar is a local phenomenon, and hence, the mode is strongly confined within the nanopillar. Using the nanopillars with diameters tuned from 80 to $210 \mathrm{~nm}$, we are able to achieve a full phase coverage of $2 \pi$, enabling complete wavefront control, while simultaneously maintaining high optical transmis$\operatorname{sion}(>70 \%)$.

To realize a metasurface capable of beam deflection, we introduce a super-cell in the $x$-direction by choosing nanopillars of diameters $124 \mathrm{~nm}, 143 \mathrm{~nm}, 167 \mathrm{~nm}$, and $207 \mathrm{~nm}$, which introduce respective phase shifts of approximately $\frac{\pi}{2}, \pi, \frac{3 \pi}{2}$, and $2 \pi$ [marked by green solid circles in Fig. 1(b)]. This supercell introduces a linear phase gradient in the $x$-direction with a periodicity of $1320 \mathrm{~nm}$. In the y-direction, the metasurface is sub-diffractive with a unit cell period of $330 \mathrm{~nm}$. The designed phase gradient will cause the metasurface to deflect a plane wave incident from the substrate into the $T_{+1}$ diffractive order. In principle, if the phase sampling is continuous (a)

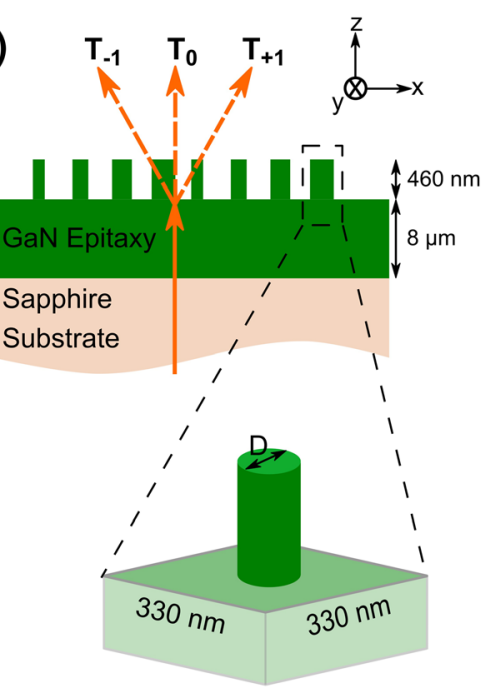

(b)

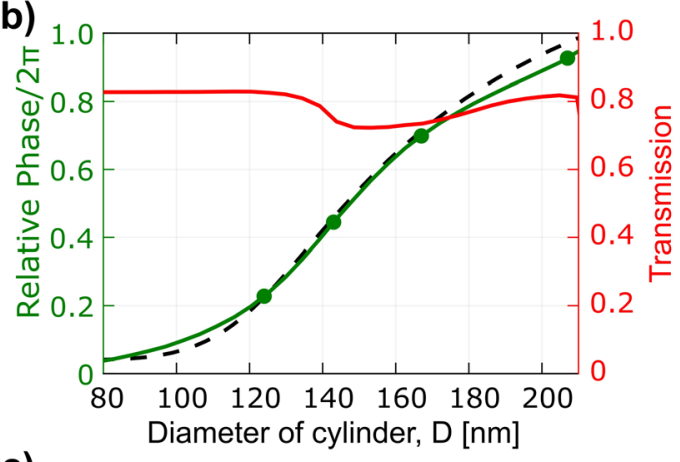

(c)

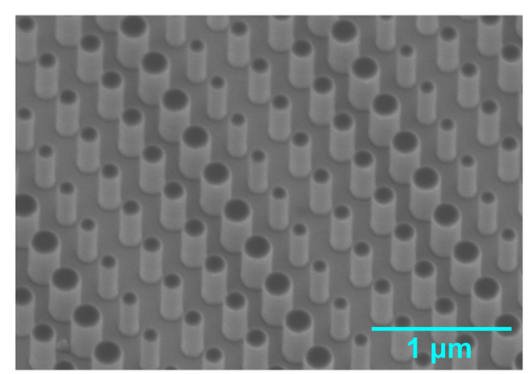

FIG. 1. (a) Schematic illustration of the proposed metasurface capable of deflecting the incident beam from the substrate into the $\mathrm{T}_{+1}$ direction. The substrate dimensions, height, and sizes of designed nanopillars are as shown. The diameter $\mathrm{D}$ was varied from 80 to $210 \mathrm{~nm}$ to realize a linear phase gradient between 0 and $2 \pi$. (b) Numerical calculations of the relative phase shift introduced by the nanopillars and transmission for a plane wave with $460 \mathrm{~nm}$ wavelength, incident from the substrate side. The dashed black curve is the analytical calculation of the phase shift introduced by an isolated cylinder. (c) SEM image of the fabricated $\mathrm{GaN}$ sample. 
and the transmission is constant, it is possible to achieve $100 \%$ deflection efficiency ${ }^{34,35}$ - meaning that there is negligible power in $\mathrm{T}_{0}$ and $\mathrm{T}_{-1}$ orders at the operating wavelength.

However, the 4-level discretization, which we chose to use here, limits the theoretical absolute efficiency of beam deflection into the first order to $\sim 81 \% .^{36}$ We should also note that in our present system, since the metasurface is of the same material as the underlying epitaxial substrate, the resulting diffraction into the substrate cannot be avoided. This can be expected to result in a further reduction in the diffraction efficiency.

The sample as described above was fabricated using standard e-beam lithography and inductively coupled reactive ion etching processes (see supplementary material A1 for additional details). A representative scanning electron microscopy (SEM) image of the metasurface studied in this work is shown in Fig. 1(c). The sample was characterized by illuminating it using a halogen lamp under normal incidence through the substrate and collecting the back-focal plane image (with an input slit) using a CCD camera (see supplementary material A2 for additional details). The images captured on the CCD show spectral and k-dependence of the energy distribution in various diffractive orders. ${ }^{37,38}$ The results for the $p$-polarization (electric field along the long period of the super-cell) are shown in Fig. 2(a). The white dashed lines represent the expected diffraction orders (in air) for our design. Clearly, most of the incident light is deflected into the $T_{+1}$ order with the deflection angle dependent on the operating wavelength as expected from a diffractive design. Figure 2(b) shows the measured diffraction intensity normalized to the transmitted intensity through the substrate. These curves are obtained by averaging five image pixels on either side of the diffraction orders depicted as white dashed lines in Fig. 2(a) (the number of pixels is selected to fully integrate the energy going into each individual diffraction order at the image). The corresponding FDTD simulations are shown as dashed curves. Figure 2(c) shows the relative efficiency, which is defined as the ratio of intensity in the desired diffraction order to the total transmitted intensity, reaching about $90 \%$ at the design wavelength of $460 \mathrm{~nm}$ where the deflection angle is $20^{\circ}$. The corresponding measured and simulated data for the $s$-polarization (electric field perpendicular to the long period of the supercell) are shown in Figs. 2(d)-2(f). The experimental measurements correspond closely to the simulations and show a peak transmission efficiency of $\sim 70 \%$ for both the $s$ - and $p$-polarizations. The transmission into the $\mathrm{T}_{0}$ and $\mathrm{T}_{-1}$ orders is quite small and is limited to about $6 \%$ and $1 \%$, respectively. The polarization insensitive behavior of our device is not surprising given the circular cross-section of the nanopillar design. The main features predicted by the numerical simulations are well reproduced in the experiment. Small discrepancies related to the absence in experiment of sharp spectral features predicted by simulations around $440 \mathrm{~nm}$ can be attributed to unavoidable nanofabrication imperfections in sidewall profiles and corner rounding, which are different for nanopillars of varying dimensions.

To show the versatility of the proposed GaN platform, we now demonstrate a metasurface with the polarization beam splitting functionality. A polarization selective metasurface can be realized by replacing the circular nanopillar by an elliptical nanopillar, wherein the phase velocity of the mode is dependent on the orientation of the input polarization with respect to the major axis of the ellipse. Here, we design and experimentally show a polarization beam splitting metasurface that deflects the $p$-polarized incoming light into the $\mathrm{T}_{+1}$ diffractive order and the $s$-polarization into the
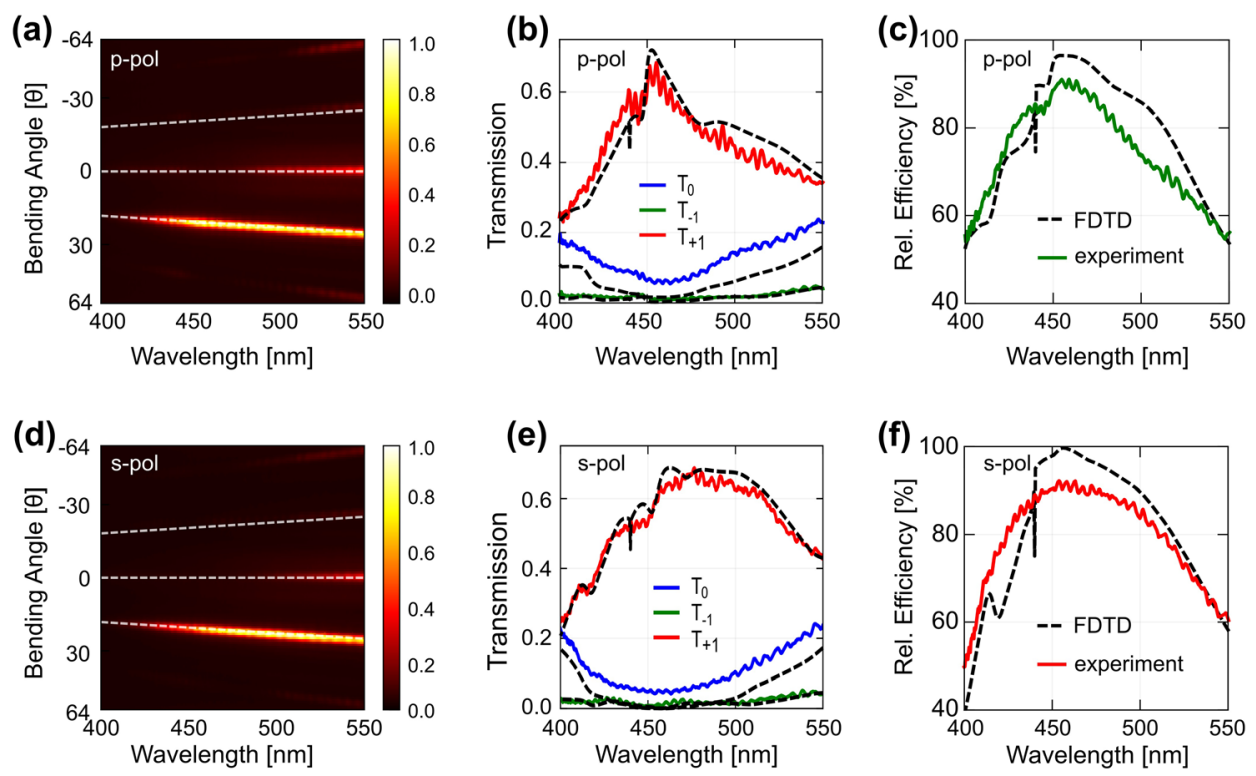

FIG. 2. Measured energy distribution into different diffraction orders as a function of the wavelength for a beam deflecting metasurface illuminated by the p-polarized (a), (b), and (c) and $s$-polarized (d), (e), and (f) light through the substrate. The transmitted light is predominantly bent into the $\mathrm{T}_{+1}$ order, with negligible intensity in the $\mathrm{T}_{0}$ and $\mathrm{T}_{-1}$ orders at the operating wavelength of $460 \mathrm{~nm}$. The white dashed lines in (a) and (d) represent the diffraction orders into air calculated for the supercell period of $1320 \mathrm{~nm}$. The color bar in (a) and (d) represents the transmitted intensity normalized to incident light at each wavelength. The experimental data (b) and (e) are obtained by averaging five pixels on either side of the diffracted orders (the white dashed lines) normalized to the substrate transmission (the number of pixels is selected to fully integrate the energy going into each individual diffraction order at the image). The black dashed curves are the simulated results, which closely match the experimental trends. Relative efficiency (c) and (f), defined as the transmitted intensity into the desired diffraction order normalized to the total transmitted intensity, reaches the level of $\sim 90 \%$ at the operation wavelength. 
(a)

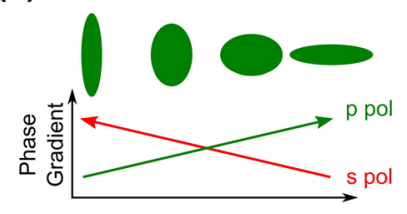

(b)

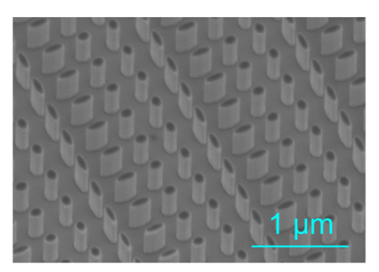

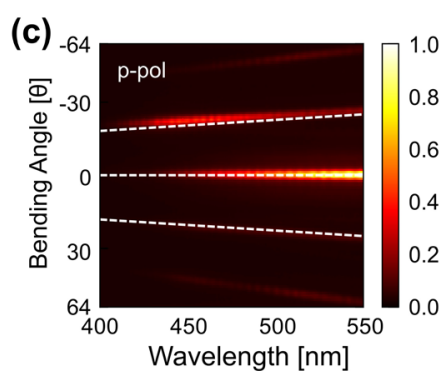

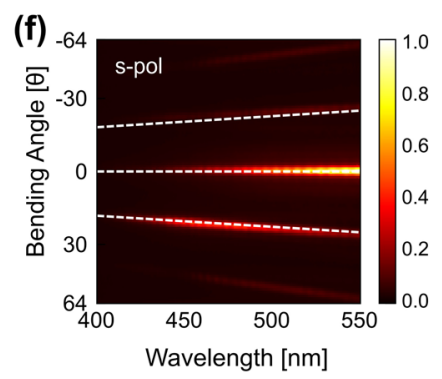

(d)

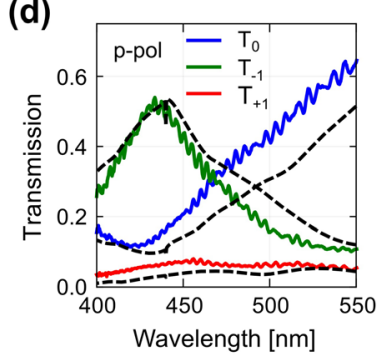

(g)

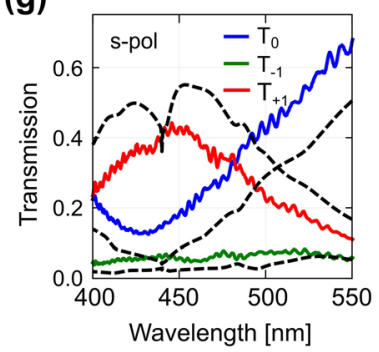

(e)

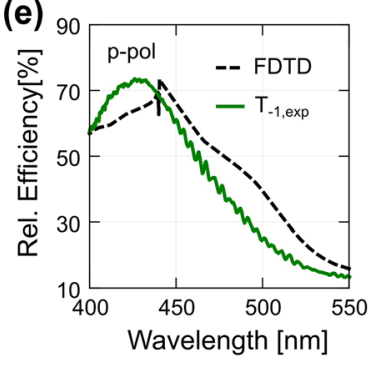

(h)

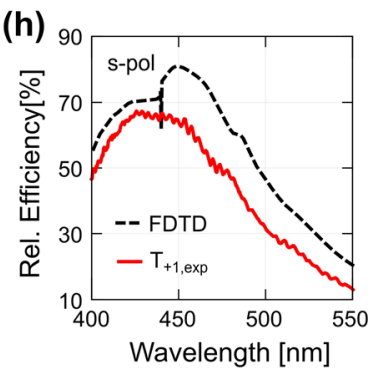

FIG. 3. (a) Schematic illustration of the phase gradients employed to demonstrate polarization beam splitting metasurface. The phase introduced by each nanopillar is dependent on the radii and the orientation relative to the polarization direction. (b) A representative SEM image of the fabricated GaN device. (c) and (f) Spectrally resolved back focal plane images showing the intensity of light transmitted in various diffraction orders for the $p$ - and $s$-polarizations, respectively. For the $p$-polarized illumination, the transmitted light deflects predominantly into the $\mathrm{T}_{-1}$ diffraction order, while for the $s$-polarized illumination, the light is directed into the $\mathrm{T}_{+1}$ order. (d) and (g) Spectral dependence of intensity in the $\mathrm{T}_{-1}, \mathrm{~T}_{0}$, and $\mathrm{T}_{+1}$ diffraction orders for the $p$ - and $s$-polarizations, respectively. (e) and (h) Relative efficiencies of light channeling into the $\mathrm{T}_{+1}$ and $\mathrm{T}_{-1}$ orders, for the $p$ - and $s$-polarizations, respectively. The measured peak relative efficiencies of beam deflection are $73 \%$ for the $p$-polarization and $67 \%$ for the $s$-polarization at $430 \mathrm{~nm}$ illumination. The solid colored curves represent the measured values, while the black dashed ones correspond to the numerical simulations.

$\mathrm{T}_{-1}$ diffractive order. The design principles are similar to the phase gradient concepts discussed earlier with one major difference-the ellipses in the supercell are arranged such that the phase gradients point in opposite directions for the $p$ - and $s$-polarizations, as schematically shown in Fig. 3(a). The amplitude transmission coefficient and phase maps obtained by varying the radii of elliptical nanopillars and the specific design parameters used are given in the supplementary material (A3). The design height was kept fixed at $460 \mathrm{~nm}$ similar to the beam deflecting metasurface above. A representative SEM image of the fabricated GaN metasurface sample is shown in Fig. 3(b). The back focal plane measurements shown in Figs. 3(c) and 3(f) demonstrate the input light deflecting to the $\mathrm{T}_{-1}$ and $\mathrm{T}_{+1}$ orders for the $p$ - and $s$-polarizations, respectively. The spectral dependence of the measured diffraction orders, along with the corresponding numerical simulations, is shown in Figs. 3(d) and 3(g). Experimentally, we measure $\sim 50 \%$ of the transmitted light channeled into the $\mathrm{T}_{-1}$ order for the $p$-polarized light and $\sim 40 \%$ into the $\mathrm{T}_{+1}$ order for the $s$-polarized light. The relative diffraction efficiencies achieved in our experiments are $\sim 74 \%$ for the $p$-polarized light and $\sim 66 \%$ for the $s$-polarized light [Figs. 3(e) and 3(h)]. The experimentally realized extinction ratios are 8.6/1 and 6.2/1 for the $p$ - and $s$-polarizations, respectively.

In conclusion, we experimentally demonstrate $\mathrm{GaN}$ as a suitable material platform for realizing a wide range of high-efficiency metasurface-based devices with enhanced functionalities operating through the whole visible spectrum including the deep blue spectral region around $450 \mathrm{~nm}$. As a proof-of-concept demonstration, we have experimentally showed an epitaxially grown $\mathrm{GaN}$ based polarization insensitive metasurface that diffracts incoming light at $460 \mathrm{~nm}$ wavelength to an angle of $20^{\circ}$ with $\sim 70 \%$ absolute transmission efficiency and $\sim 90 \%$ relative transmission efficiency. These reasonably high efficiencies are achieved despite the fact that the refractive index of the metasurface is the same as the underlying substrate, which is widely believed to lower the efficiency. Additionally, we have also demonstrated a polarization beam splitter working at $430 \mathrm{~nm}$ wavelength and capable of separating the $p$ - and $s$-polarizations with the relative efficiencies of $73 \%$ and $67 \%$, respectively. The corresponding extinction ratios of 8.6/1 and 6.2/1 for the $p$ - and $s$ - polarizations, respectively, were obtained. We expect that further development of metasurfaces based on $\mathrm{GaN}$ and its alloys with $\mathrm{InN}$ and AlN will pave the way for active, nonlinear, and quantum nanophotonics compatible with the emission wavelengths of atomic quantum emitters such as $\mathrm{Ca}^{+}$and $\mathrm{Sr}^{+}$ions. ${ }^{39}$

See supplementary material for a complete description of the nanofabrication and optical characterization methods and design of the polarizing beam splitter. 
This research was financially supported by A*STAR SERC Pharos program (Grant No. 15273 00025). Fabrication and Scanning Electron Microscope imaging works were carried out at the SnFPC cleanroom facility at Data Storage Institute (SERC Grant No. 092160 0139).

${ }^{1}$ N. Yu, P. Genevet, M. A. Kats, F. Aieta, J.-P. Tetienne, F. Capasso, and Z. Gaburro, Science 334(6054), 333 (2011).

${ }^{2}$ N. Yu, F. Aieta, P. Genevet, M. A. Kats, Z. Gaburro, and F. Capasso, Nano Lett. 12(12), 6328 (2012).

${ }^{3}$ S. Ishii, A. V. Kildishev, V. M. Shalaev, K.-P. Chen, and V. P. Drachev, Opt. Lett. 36(4), 451 (2011).

${ }^{4}$ O. Akın and H. V. Demir, Appl. Phys. Lett. 110(14), 143106 (2017).

${ }^{5}$ A. Pors, M. G. Nielsen, R. L. Eriksen, and S. I. Bozhevolnyi, Nano Lett. 13(2), 829 (2013).

${ }^{6}$ X. Ni, A. V. Kildishev, and V. M. Shalaev, Nat. Commun. 4, 2807 (2013).

${ }^{7}$ N. Yu and F. Capasso, Nat. Mater. 13(2), 139 (2014).

${ }^{8}$ F. Ding, A. Pors, and S. I. Bozhevolnyi, "Gradient metasurfaces: a review of fundamentals and applications," Rep. Prog. Phys. (to be published).

${ }^{9}$ J. B. Khurgin and A. Boltasseva, MRS Bull. 37(08), 768 (2012).

${ }^{10}$ A. I. Kuznetsov, A. E. Miroshnichenko, M. L. Brongersma, Y. S. Kivshar, and B. Luk'yanchuk, Science 354(6314), aag2472 (2016).

${ }^{11}$ M. Decker, I. Staude, M. Falkner, J. Dominguez, D. N. Neshev, I. Brener, T. Pertsch, and Y. S. Kivshar, Adv. Opt. Mater. 3(6), 813 (2015).

${ }^{12}$ D. Lin, P. Fan, E. Hasman, and M. L. Brongersma, Science 345(6194), 298 (2014).

${ }^{13}$ R. C. Devlin, M. Khorasaninejad, W. T. Chen, J. Oh, and F. Capasso, in Proceedings of the National Academy of Sciences (2016), p. 201611740.

${ }^{14}$ E. Maguid, I. Yulevich, D. Veksler, V. Kleiner, M. L. Brongersma, and E. Hasman, Science 352(6290), 1202 (2016).

${ }^{15}$ M. Khorasaninejad, W. T. Chen, R. C. Devlin, J. Oh, A. Y. Zhu, and F. Capasso, Science 352(6290), 1190 (2016).

${ }^{16}$ A. Arbabi, Y. Horie, M. Bagheri, and A. Faraon, Nat. Nanotechnol. 10, 937 (2015).

${ }^{17}$ M. Khorasaninejad, A. Y. Zhu, R.-C. Charles, W. T. Chen, J. Oh, I. Mishra, R. C. Devlin, and F. Capasso, Nano Lett. 16(11), 7229 (2016).

${ }^{18}$ A. Arbabi, E. Arbabi, S. M. Kamali, Y. Horie, S. Han, and A. Faraon, Nat. Commun. 7, 13682 (2016).
${ }^{19}$ P. R. West, J. L. Stewart, A. V. Kildishev, V. M. Shalaev, V. V. Shkunov, F. Strohkendl, Y. A. Zakharenkov, R. K. Dodds, and R. Byren, Opt. Express 22(21), 26212 (2014).

${ }^{20}$ P. Lalanne and P. Chavel, Laser Photonics Rev. 11(3), 1600295 (2017).

${ }^{21}$ D. G. Baranov, D. A. Zuev, S. I. Lepeshov, O. V. Kotov, A. E. Krasnok, A. B. Evlyukhin, and B. N. Chichkov, Optica 4(7), 814 (2017).

${ }^{22}$ A. Zhan, S. Colburn, R. Trivedi, T. K. Fryett, C. M. Dodson, and A. Majumdar, ACS Photonics 3(2), 209 (2016).

${ }^{23}$ S. Liu, G. A. Keeler, J. L. Reno, M. B. Sinclair, and I. Brener, Adv. Opt. Mater. 4(10), 1457 (2016).

${ }^{24}$ M. R. Shcherbakov, S. Liu, V. V. Zubyuk, A. Vaskin, P. P. Vabishchevich, G. Keeler, T. Pertsch, T. V. Dolgova, I. Staude, and I. Brener, Nat. Commun. 8(1), 17 (2017).

${ }^{25}$ Y. Yang, N. Kamaraju, S. Campione, S. Liu, J. L. Reno, M. B. Sinclair, R. P. Prasankumar, and I. Brener, ACS Photonics 4, 15-21 (2017).

${ }^{26}$ J. Cambiasso, G. Grinblat, Y. Li, A. Rakovich, E. Cortés, and S. A. Maier, Nano Lett. 17(2), 1219 (2017).

${ }^{27}$ I. A. Walmsley, Science 348(6234), 525 (2015).

${ }^{28}$ S. Deshpande, J. Heo, A. Das, and P. Bhattacharya, Nat. Commun. 4, 1675 (2013).

${ }^{29}$ M. J. Holmes, K. Choi, S. Kako, M. Arita, and Y. Arakawa, Nano Lett. 14(2), 982 (2014).

${ }^{30}$ Z. Wang, S. He, Q. Liu, and W. Wang, Opt. Commun. 367, 144 (2016).

${ }^{31}$ B. H. Chen, P. C. Wu, V.-C. Su, Y.-C. Lai, C. H. Chu, I. C. Lee, J.-W. Chen, Y. H. Chen, Y.-C. Lan, C.-H. Kuan, and D. P. Tsai, Nano Lett. 17(10), 6345 (2017).

${ }^{32}$ S. P. DenBaars, D. Feezell, K. Kelchner, S. Pimputkar, C.-C. Pan, C.-C. Yen, S. Tanaka, Y. Zhao, N. Pfaff, and R. Farrell, Acta Mater. 61(3), 945 (2013).

${ }^{33}$ A. W. Snyder and J. Love, Optical Waveguide Theory (Springer Science \& Business Media, 2012).

${ }^{34}$ V. S. Asadchy, M. Albooyeh, S. N. Tcvetkova, A. Díaz-Rubio, Y. Ra'di, and S. A. Tretyakov, Phys. Rev. B 94(7), 075142 (2016).

${ }^{35}$ N. M. Estakhri and A. Alù, Phys. Rev. X 6(4), 041008 (2016).

${ }^{36}$ G. J. Swanson, MIT Tech. Report No. 854, 1989.

${ }^{37}$ R. Paniagua-Dominguez, Y. F. Yu, E. Khaidarov, R. M. Bakker, X. Liang, Y. H. Fu, and A. I. Kuznetsov, preprint arXiv:1705.00895 (2017).

${ }^{38}$ E. Khaidarov, H. Hao, R. Paniagua-Dominguez, Y. Yu, Y. H. Fu, V. Valuckas, S. L. K. Yap, Y. T. Toh, J. S. K. Ng, and A. I. Kuznetsov, Nano Lett. 17(10), 6267 (2017).

${ }^{39}$ H. Häffner, C. F. Roos, and R. Blatt, Phys. Rep. 469(4), 155 (2008). 